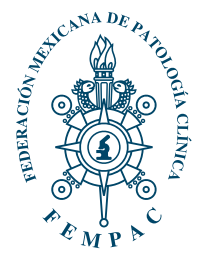

\title{
El rol del patólogo clínico en COVID-19. Una perspectiva peruana
}

\author{
The role of the clinical pathologist in COVID-19. A Peruvian perspective
}

\author{
La Rosa Fabián Christian Blas,* Urquizo Briceño Leticia ${ }^{\ddagger}$
}

\author{
Palabras clave: \\ SARS-CoV-2, \\ COVID-19, patólogo \\ clínico. \\ Keywords: \\ SARS-COV-2, \\ COVID-19, clinical \\ pathologist.
}

\begin{abstract}
* Departamento de Patología Clínica y Anatomía Patológica. Facultad de Medicina. ‡ Médico Residente de Patología Clínica.
\end{abstract}

Hospital Nacional Dos de Mayo. Universidad Nacional Mayor de San Marcos. Lima, Perú.

Correspondencia:

Dr. Christian Blas La

Rosa Fabián

Departamento de

Patología Clínica y Anatomía Patológica. Hospital Nacional Dos de Mayo.

Parque «Historia de la Medicina Peruana» $\mathrm{s} / \mathrm{n}$,

Cercado de LimaLima, Perú.

E-mail: chris_blas@ hotmail.com

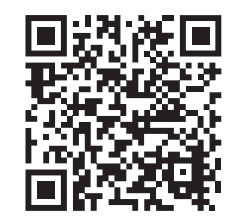

\section{RESUMEN}

El virus SARS-CoV-2, el agente etiológico del COVID-19, fue descubierto recientemente en medio de una pandemia que ha resultado en una gran morbilidad y mortalidad a nivel mundial con un gran costo económico y social. La patología clínica como especialidad otorga al médico la capacidad de ayudar a llegar a un diagnóstico a través de pruebas de laboratorio, monitorizar y controlar la evolución de las enfermedades, evaluar el pronóstico de los pacientes, gestionar el laboratorio clínico, desarrollar actividades de investigación y docencia con el fin de obtener una mejor comprensión de las enfermedades y sus consecuencias en el cuerpo humano. En el contexto de la pandemia de COVID-19, el patólogo clínico tiene un papel importante en estas áreas. Si bien la ayuda diagnóstica de laboratorio más evidente ha sido la detección del virus SARS-CoV-2 mediante pruebas moleculares, el alcance de la medicina de laboratorio va más allá, constituyendo una herramienta fundamental en el manejo y control de la enfermedad.

\section{ABSTRACT}

The SARS-CoV-2 virus, the etiological agent of COVID-19, was discovered recently, in the midst of a pandemic that has resulted in great morbidity and mortality worldwide, at great economic and social cost. Clinical pathology as a specialty gives the physician the ability to help reach a diagnosis through laboratory tests, monitor and control the evolution of diseases, evaluate the prognosis of patients, manage the clinical laboratory, develop research and teaching activities in order to gain a better understanding of diseases and their consequences in the human body. In the context of the COVID-19 pandemic, the clinical pathologist plays an important role in these areas. Although the most obvious laboratory diagnostic aid has been the detection of the SARSCoV-2 virus through molecular tests, the scope of laboratory medicine goes further, constituting a fundamental tool in the management and control of the disease.

\section{INTRODUCCIÓN}

A finales de 2019, la ciudad de Wuhan, ubicada en la provincia china de Hubei, se convirtió en el epicentro de un brote de una neumonía de causa desconocida. Posteriormente se confirmó que la neumonía era causada por un nuevo coronavirus. Este virus se llamó originalmente el nuevo coronavirus 2019 (2019-nCoV), pero el 11 de febrero de 2020 la Organización Mundial de la Salud (OMS) denominó a la enfermedad COVID-19 y al virus causante del síndrome respiratorio agudo severo (SARS-CoV-2) se le llamó coronavirus de tipo 2 por el Comité Internacional de
Taxonomía de Virus (ICTV) y dicha enfermedad fue considerada pandemia por la OMS el 11 de marzo de $2020 .^{1,2}$

En el Perú, el 6 de marzo de 2020 se confirmó a la primera persona infectada y se incrementó rápidamente el número de casos sospechosos, para el 19 de marzo se presentaron las tres primeras víctimas mortales. ${ }^{3}$

A un gran costo económico, muchos países como Perú han adoptado medidas sin precedentes para frenar la propagación del virus como el uso a gran escala del aislamiento y la cuarentena, el cierre de fronteras, la imposición de límites a las reuniones públicas y la implementación de bloqueos a nivel nacional. ${ }^{4}$

Citar como: La Rosa FCB, Urquizo BL. El rol del patólogo clínico en COVID-19. Una perspectiva peruana. Rev Mex Patol Clin Med Lab. 2020; 67 (4): 185-189. https://dx.doi.org/10.35366/99465 
Con base principalmente en datos de China, alrededor de $80 \%$ de las personas infectadas con SARS-CoV-2 experimentan un «resfriado» relativamente leve como se observa con las infecciones por coronavirus más comunes. Sin embargo, 20\% de los infectados requieren hospitalización y $5-15 \%$ en general necesita cuidados intensivos. ${ }^{5}$ Por lo tanto, el diagnóstico temprano y el tratamiento oportuno son cruciales.

En octubre de 2020, Perú tenía 27,146 médicos activos distribuidos en 70 especialidades médicas reconocidas por el Colegio Médico del Perú (CMP). De éstos, 760 son patólogos clínicos con título de especialista. Además, se ofrecen cada año aproximadamente 2,188 vacantes de residencia médica para 46 especialidades y 40 subespecialidades, de las cuales 58 vacantes son para patología clínica. ${ }^{6}$

El patólogo clínico utiliza su formación clínica y conocimiento de la medicina de laboratorio para ayudar al médico tratante con respecto a la prevención, diagnósti$\mathrm{Co}$, tratamiento, monitoreo terapéutico, seguimiento de enfermedades y pronóstico. Establece una interfaz con otras especialidades médicas, permitiendo discutir las implicaciones clínicas de los resultados y sugerir métodos propedéuticos alternativos para una situación dada, convirtiéndolo en un verdadero consultor. Es el profesional que tiene el conocimiento necesario para una comunicación bidireccional al mismo nivel que el médico tratante porque posee la misma formación médica.

El patólogo clínico, así como el resto de las especialidades, se enfrentó a un nuevo virus que fuimos aprendiendo durante la pandemia, una nueva enfermedad con características diferentes a lo producido por otros coronavirus, una fisiopatología con muchos aspectos hasta ahora desconocidos con afectación a varios sistemas. El laboratorio se enfrentó, entre otras cosas, a pruebas nuevas, no se tenía conocimiento de su desempeño, interferencia y evolución como marcadores.

Para un médico que no tiene un conocimiento a profundidad del laboratorio, puede ser más complicado realizar una interpretación de los resultados, el patólogo clínico al saber cómo se produce el resultado, tiene mayor facilidad para la interpretación y cuál es la mejor conducta a seguir frente a dicho resultado. A pesar del tiempo, en estos exámenes aún siguen existiendo muchas preguntas sin respuestas.

En este escenario de desafío frente a la pandemia COVID-19 el patólogo clínico tiene un importante papel, es pieza clave en la asistencia del apoyo diagnóstico, pero no sólo en el razonamiento diagnóstico, sino también en su contribución con pruebas necesarias para el monitoreo, pronóstico, ayuda en la toma de decisiones terapéuticas y en la conducción de una herramienta terapéutica como el uso del plasma convaleciente (Figura 1).

\section{DIAGNÓSTICO}

El entorno más obvio en el que el laboratorio desempeña un papel esencial es el diagnóstico etiológico del COVID-19. Es importante poder guiar al médico tratante en el acceso adecuado a las pruebas de diagnóstico apropiadas según las recomendaciones existentes a nivel mundial, y resolver la confusión entre los profesionales de la salud y el público sobre la priorización de las pruebas y la interpretación de los resultados.

El estándar de oro actual para diagnosticar los casos sospechosos de COVID-19 es la detección de secuencias
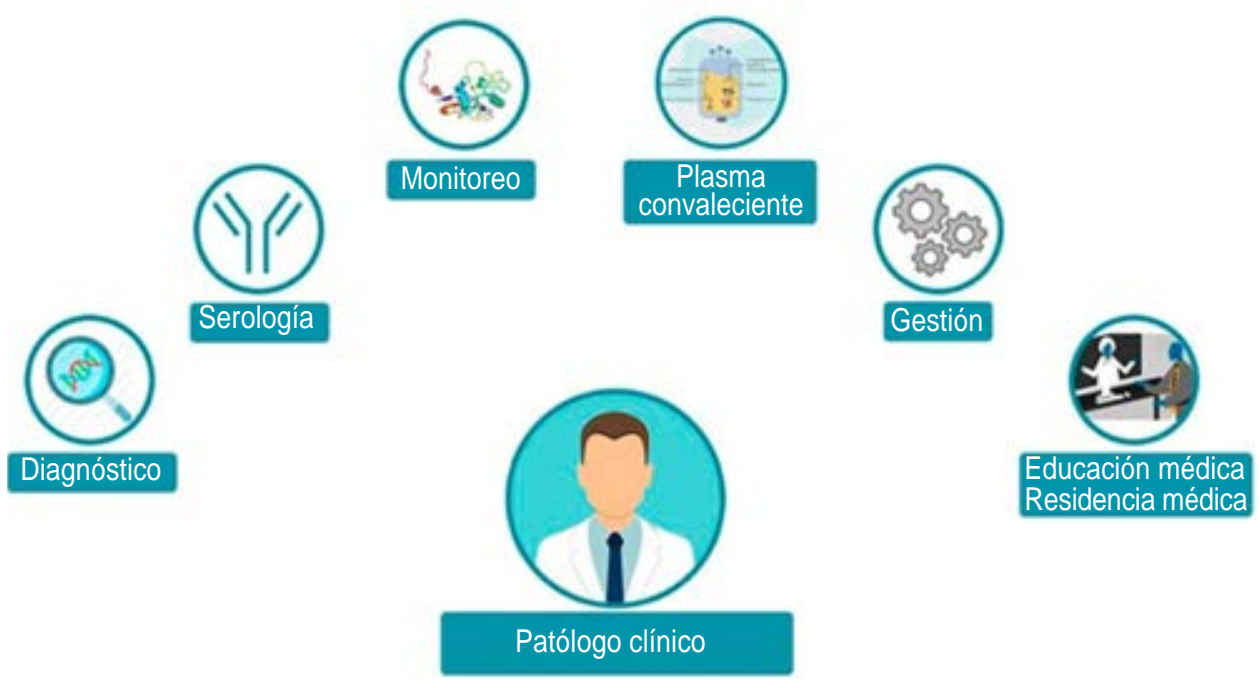

Figura 1:

Papel del patólogo clínico en el COVID-19. 
únicas de ARN viral por la reacción en cadena de polimerasa de transcripción inversa en tiempo real (rRT-PCR), pero es necesario evaluar el rendimiento diagnóstico de las muestras, los límites de detección de los métodos realizados y cuáles son las muestras recomendadas. Asimismo, poder tener la visión integral de todas las fases del laboratorio (preanalítica, analítica y postanalítica) con el fin de evaluar los falsos negativos y falsos positivos que es una de las principales consultas en la práctica diaria. ${ }^{7,8}$

Por eso, un paso crucial es la selección del ensayo para la implementación de la prueba molecular, la cual debe considerar metodología, diana de genes, rendimiento/muestra, factibilidad de verificación de ensayos y limitaciones, todo esto asociado con la accesibilidad de los kits comerciales y la instrumentación complementaria necesaria. ${ }^{9,10}$

Un claro enfoque de la calidad sigue siendo de gran importancia para proporcionar resultados precisos e interpretables, por ejemplo, el desarrollo de un programa de garantía de calidad como el realizado por el Real Colegio de Patólogos de Australasia (RCPAQAP). ${ }^{11}$

\section{SEROLOGÍA}

Otro pilar importante es el uso de pruebas serológicas para medir la respuesta de anticuerpos al virus, que en determinadas circunstancias son complementarias para el diagnóstico y toman un gran valor para refinar la compresión de la intensidad de transmisión y la susceptibilidad de la población. ${ }^{12}$

El patólogo clínico debe orientar al uso correcto de estas pruebas en la atención médica; tiene que conocer las características relacionadas con la serología como la gran variedad de plataformas disponibles que incluyen inmunoensayos clásicos de fase sólida como el ensayo por inmunoabsorción ligado a enzimas (ELISA), métodos basados en perlas, citometría de flujo, quimioluminiscencia y los ensayos inmunocromatográficos de flujo lateral de gran interés debido a su uso en el punto de atención del paciente (POC); además, dicho especialista evaluará las características de rendimiento de las pruebas entre las diferentes plataformas, la diana antigénica, la clase de anticuerpo detectado: $\lg \mathrm{A}$, $\lg \mathrm{M}$, $\lg \mathrm{G}$ o anticuerpos totales que son muy importantes y de gran desafío, en los que se plantean problemas en relación con los umbrales de sensibilidad/especificidad, reacción cruzada con otros coronavirus particularmente con los del subgrupo B, el uso de ensayos de neutralización como referencia de estándar de oro, dificultades para armonizar informes de resultados, preocupaciones por el control de calidad en la fabricación, y lo más importante una falta de línea de base requerida para la interpretación de la prueba. ${ }^{13-15}$
Por ejemplo, los ensayos inmunocromatográficos de flujo lateral llamados comúnmente «prueba rápida» al parecer tienen más problemas de sensibilidad y especificidad que las otras metodologías; ${ }^{16,17}$ sin embargo, son de bajo costo, fáciles de usar y permiten una ampliación a gran escala y adopción generalizada. Una estrategia alternativa es centralizar las pruebas en laboratorios clínicos mediante el uso de inmunoensayos totalmente automatizados, aunque es más costoso, incluye mayor precisión y confiabilidad, la posibilidad de generar datos cuantitativos (que son esenciales para el monitoreo longitudinal de los títulos), el desempeño por parte de personal calificado de laboratorio (reduciendo así inherentemente el riesgo de errores e interpretación subjetiva), almacenamiento permanente de la prueba, resultados dentro del sistema de información de laboratorio (LIS), junto con un monitoreo de calidad más estricto según lo permita el desempeño del control de calidad interno $y$, con suerte en un futuro cercano, esquemas de evaluación de calidad externa (EQA).

\section{MONITOREO}

Tan importante como el diagnóstico es el seguimiento de pacientes con COVID-19 en el ambiente hospitalario, la gravedad de la enfermedad puede sugerirse y notificarse al médico tratante con conocimiento mayor de la fisiopatología de la enfermedad y con base en numerosos estudios que identifican las anormalidades asociadas en exámenes de laboratorio.

El médico patólogo clínico asume un papel de asesoría en el sentido de que orienta al clínico sobre qué exámenes solicitar en función de la historia de la enfermedad y la situación clínica del paciente. Este papel está precedido de un trabajo constante de actualización e investigación en medicina de laboratorio. El estudio del comportamiento de diferentes biomarcadores en COVID-19 ha dado luces sobre su utilidad en la predicción de la evolución a estadios graves de la enfermedad, daño a órganos específicos y monitoreo de respuesta al tratamiento. Podemos mencionar dentro de un examen básico de hemograma, que el recuento de linfocitos es un parámetro de obtención rápida y comúnmente accesible, importante para poder discriminar pacientes con enfermedad grave. ${ }^{18}$ Además, tenemos biomarcadores que han cobrado una mayor relevancia como la interleucina 6 (IL-6), importante citoquina proinflamatoria, útil como marcador pronóstico; la ferritina, predictor de enfermedad grave; y el dímero $\mathrm{D}$, asociado con un peor pronóstico en pacientes con enfermedad severa. ${ }^{19,20}$

Ya sea en la medición de marcadores de inflamación como en la evaluación de las alteraciones hematológicas y bioquímicas, el laboratorio desempeña un papel cru- 
cial en el seguimiento de los pacientes con COVID-19, por lo que el médico patólogo clínico debe garantizar exámenes confiables y precisos que sigan metodologías adecuadas y salvaguardar que los resultados lleguen al médico tratante de manera oportuna para una toma de decisiones adecuada.

\section{BANCO DE SANGRE-PLASMA CONVALECIENTE}

Los servicios de banco de sangre han tenido que responder de la manera más adecuada, trabajando diligentemente para realizar cambios frecuentes en los planes a medida que se desarrollaba la pandemia, promoviendo la donación de sangre comunitaria, aumentando las actividades de recolección en los hospitales, solicitando reducir las operaciones hospitalarias o al menos advirtiendo que la sangre se estaba convirtiendo rápidamente en un recurso muy escaso. ${ }^{21}$

El uso de plasma convaleciente (PC) ha surgido como opción terapéutica ante la ausencia de una terapia antiviral específica. Existen estudios con resultados óptimos para esta terapia, ${ }^{22,23}$ lo que alienta a desarrollar nuevos estudios clínicos que aporten mayor data sobre este método.

La Food and Drug Administration (FDA) de los Estados Unidos ha publicado una guía para el uso de plasma convaleciente durante el estado de emergencia sanitaria. En ésta se establecen las condiciones que deben cumplir los bancos de sangre que realicen la colección y distribución de PC. Con la finalidad de cumplir los requisitos para la selección de donantes de PC, el patólogo clínico tiene un papel esencial en la ejecución de este proceso, ya que puede guiar un interrogatorio adecuado con sus conocimientos de clínica médica y tiene la capacidad de evaluar la pertinencia del resultado positivo para COVID-19 que se requiere (en cuanto a tiempo de enfermedad al momento de llevar a cabo el examen y metodología empleada). La implementación de pruebas de determinación de anticuerpos tipo IgG por ELISA o quimioluminiscencia es otro requisito para resolver si el plasma recolectado es apto para donación así como su titulación. Los subsiguientes procesos de la cadena transfusional, que son comunes a las transfusiones de plasma ya conocidos, no están eximidos de cumplir con los requisitos de calidad y seguridad propios de los procedimientos terapéuticos ofrecidos por las unidades de hemoterapia.

\section{GESTIÓN}

El patólogo clínico no sólo cumple con su papel asistencial, sino también con el de gestor, que ha permitido que los laboratorios clínicos alcancen las metas trazadas por su misión, visión institucional e implementación, ciñéndose a las recomendaciones de las guías nacionales e internacionales, a la constante actualización en cuanto a metodologías, equipos y flujos de trabajo y adecuándose a la demanda de servicios de salud en pos de brindar resultados precisos y confiables.

En el contexto del COVID-19, los laboratorios han tenido que adaptarse a los requerimientos de exámenes de diagnóstico y seguimiento de los pacientes con sospecha o ya diagnosticados con la enfermedad. En ese sentido, las unidades proveedoras de servicio de patología clínica han reajustado su oferta, ya que los servicios de consultorio externo y hospitalización por otras patologías se vieron suspendidas o limitadas al inicio de la contingencia sanitaria, por lo que muchos exámenes como hormonas, estudios de fertilidad, de seguimiento de enfermedades crónicas fueron dejados de lado para dar paso a la medición de marcadores de inflamación y de trombosis, un aumento en el volumen de hemogramas a analizar y la reciente implementación de laboratorios de biología molecular descentralizados, con el fin de no sólo dar un buen resultado en el aspecto cuantitativo o cualitativo, sino para mejorar los tiempos de respuesta en que éstos llegan al médico tratante.

A lo largo de la pandemia los jefes y gerentes de laboratorio han adaptado diferentes medidas para hacer frente a la falta de reactivos por el incremento de demanda, el cambio de algunos reactivos (pruebas suspendidas) por fecha de vencimiento próxima con los proveedores, ya que los requerimientos no avizoraban la contingencia sanitaria en el momento en que se realizaron, la implementación de protocolos de bioseguridad en uso de equipos de protección personal (EPP), toma y conservación de muestras de origen respiratorio y manipulación de muestras biológicas por parte del personal de preanalítica y analistas. La gestión de recursos humanos en el laboratorio clínico ha constituido también un reto, se ha reducido el número de personal por descansos médicos, presencia de comorbilidades y edad avanzada, por lo que se trabaja con personal reducido ante un inminente aumento de casos y consiguiente demanda.

Esto motiva al patólogo clínico como jefe de laboratorio a tener una capacitación constante en gerencia y gestión, a estar constantemente actualizado en materia de implementación de nuevos métodos y a manejar una comunicación asertiva con proveedores y recursos humanos enfocados en la satisfacción del usuario como punto principal, recordando que es nuestro objetivo final como servicio de salud. ${ }^{24}$ 


\section{DOCENCIA}

No olvidemos que el patólogo tiene un papel docente con los médicos en formación de la especialidad, como en otras especialidades, en algunos casos ha habido reubicación de médicos residentes en salas de atención de pacientes COVID-19 así como suspensión de rotaciones. Es importante continuar de alguna manera este acompañamiento buscando alternativas como actividades virtuales, virtualización de contenidos que permitan el aprendizaje independiente, actividades de investigación, que han demostrado aumentar la motivación, el compromiso y el razonamiento cuántico y crítico necesario para obtener un adecuado desempeño en cualquier especialidad médica. ${ }^{25}$

\section{CONCLUSIONES}

Por último, el patólogo clínico, junto con las otras especialidades médicas, ha demostrado una resistencia extraordinaria en el manejo de la crisis actual con un trabajo sin miedo y una devoción total. Esta crisis ha demostrado una vez más que los diagnósticos de laboratorio siempre han sido, siguen siendo y seguirán siendo el núcleo de la toma de decisiones clínicas.

\section{REFERENCIAS}

1. Coronavirus disease 2019 (COVID-19): situation report-51. Geneva: World Health Organization; Available in: https://www.who.int/docs/ default-source/coronaviruse/situation-reports/20200311-sitrep-51covid-19.pdf.

2. Huang C, Wang Y, Li X, Ren L, Zhao J, Hu Y et al. Clinical features of patients infected with 2019 novel coronavirus in Wuhan, China. Lancet. 2020; 395 (10223): 497-506. doi: 10.1016/S01406736(20)30183-5.

3. Ministerio de Salud. Atención y manejo clínico de casos de COVID19. Escenario de transmisión focalizada. Lima-Perú; 2020.

4. Bedford J, Enria D, Giesecke J, Heymann DL, Ihekweazu C, Kobinger $\mathrm{G}$ et al. COVID-19: towards controlling of a pandemic. Lancet. 2020; 395 (10229): 1015-118. doi: 10.1016/S01406736(20)30673-5.

5. Pan F, Ye T, Sun P, Gui S, Liang B, Li L et al. Time course of lung changes at chest CT during recovery from coronavirus disease 2019 (COVID-19). Radiology. 2020; 295 (3): 715-721.

6. Pamo-Reyna OG. El residentado médico en el Perú. Diagnóstico. 2019; 58 (3): 117-121.

7. Lippi G, Simundic AM, Plebani M. Potential preanalytical and analytical vulnerabilities in the laboratory diagnosis of coronavirus disease 2019 (COVID-19). Clin Chem Lab Med. 2020; 58 (7): 1070-1076.

8. Woloshin S, Patel N, Kesselheim AS. False negative tests for SARSCoV-2 infection - challenges and implications. N Engl J Med. 2020; 383 (6): e38.

9. Carter LJ, Garner LV, Smoot JW, Li Y, Zhou Q, Saveson CJ et al. Assay techniques and test development for COVID-19 diagnosis. ACS Cent Sci. 2020; 6 (5): 591-605. doi: 10.1021/acscentsci.0c00501.

10. Li C, Zhao C, Bao J, Tang B, Wang Y, Gu B. Laboratory diagnosis of coronavirus disease-2019 (COVID-19). Clin Chim Acta. 2020; 510: 35-46. doi: 10.1016/j.cca.2020.06.045.

11. Bastian I, Waring L. The Royal College of Pathologists of Australasia (RCPA) position statement on COVID-19. Aust J Gen Pract. 2020; 49. doi: 10.31128/AJGP-COVID-09.

12. Petherick A. Developing antibody tests for SARS-CoV-2. Lancet. 2020; 395 (10230): 1101-1102. doi: 10.1016/S01406736(20)30788-1.

13. Amanat F, Stadlbauer D, Strohmeier S, Nguyen THO, Chromikova $\mathrm{V}$, McMahon $\mathrm{M}$ et al. A serological assay to detect SARS-CoV-2 seroconversion in humans. Nat Med. 2020; 26 (7): 1033-1036. doi: 10.1038/s41591-020-0913-5.

14. Theel ES, Slev P, Wheeler S, Couturier MR, Wong SJ, Kadkhoda K. The role of antibody testing for SARS-CoV-2: is there one? J Clin Microbiol. 2020; 58 (8): e00797-20.

15. Lippi G, Plebani M. SARS-CoV-2 antibodies titration: a reappraisal. Ann Transl Med. 2020; 8 (16): 1032. doi: 10.21037/atm-20-5361.

16. Lisboa Bastos M, Tavaziva G, Abidi SK, Campbell JR, Haraoui LP, Johnston JC et al. Diagnostic accuracy of serological tests for covid-19: systematic review and meta-analysis. BMJ. 2020; 370:m2516. doi: 10.1136/bmj.m2516.

17. Herroelen PH, Martens GA, De Smet D, Swaerts K, Decavele AS. Humoral immune response to SARS-CoV-2. Am J Clin Pathol. 2020; 154 (5): 610-619. doi: 10.1093/ajcp/aqaa140.

18. Ruan Q, Yang K, Wang W, Jiang L, Song J. Clinical predictors of mortality due to COVID-19 based on an analysis of data of 150 patients from Wuhan, China. Intensive Care Med. 2020; 46 (5): 846-848.

19. Coomes EA, Haghbayan H. Interleukin-6 in Covid-19: A systematic review and meta-analysis. Rev Med Virol. 2020; 30 (6): 1-9.

20. Tang N, Li D, Wang X, Sun Z. Abnormal coagulation parameters are associated with poor prognosis in patients with novel coronavirus pneumonia. J Thromb Haemost. 2020; 18 (4): 844-847.

21. Gehrie E, Tormey CA, Sanford KW. Transfusion Service Response to the COVID-19 Pandemic. Am J Clin Pathol. 2020; 154 (3): 280285. doi: 10.1093/ajcp/aqaa111.

22. Altuntas F, Ata N, Yigenoglu TN, Basci S, Dal MS, Korkmaz S et al. Convalescent plasma therapy in patients with COVID-19. Transfus Apher Sci. 2020: 102955. doi: 10.1016/j.transci.2020.102955.

23. Roback JD, Guarner J. Convalescent plasma to treat COVID-19: possibilities and challenges. JAMA. 2020; 323 (16): 1561-1562.

24. Kroft SH. The evolution of the clinical pathologist. Am J Clin Pathol. 2018; 150 (4): 283-284. doi: 10.1093/ajcp/aqy119.

25. Sánchez-Duque JA. Medical education in pandemic time: The case of coronavirus disease 2019 (COVID-19). Educación Médica. 2020; 21 (4): 259-26. doi: 10.1016/j.edumed.2020.05.005. 